\title{
SCANNING ACOUSTICAL MICROSCOPY
}

Part I: A Primer

Jacqueline Gallet, Ph.D, University of California, Irvine

One of Olympus' not well known product in the American market is the UH3 Scanning Acoustic Microscope (SAM). This state of the art, highly versatile microscope has many applications from non-destructive imaging to biomedical analysis, to pharmaceutical applications to name a few areas of current industrial interest.

The principle behind SAM is quite simple, and uses the basic physical laws of reflection. High frequency sound waves are mechanically produced by a piezoelectric crystal. A high voltage impulse spike starts the crystal vibrating at its preset resonant frequency emitting acoustical plane waves through a medium with a relatively high sound velocity such as sapphire. The waves are made to converge by a half-spherical lens at the bottom of the sapphire rod. The diameter of the lens is less than one millimeter and depends on the operating frequency. The lower the frequency, the larger is the diameter of the lens. Also, because of the high sound speed in sapphire $(11,000 \mathrm{~m} / \mathrm{s})$ spherical aberrational effects can be lessened. The lens is coated with a quarter-wavelength anti-reflective material to improve the propagation in the forward direction of the acoustical pulses. The opening angle of the lens determines the minimum resolution which can be obtained. For example, when using a $600 \mathrm{MHz}$ lens with an opening angle of 60 degrees, the minimum resolution can be no larger than 2.5 microns. When the opening angle is 120 degrees, on the other hand, the minimum

\section{Desktop
Microsco
forthacintosto}

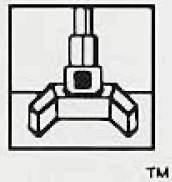

M
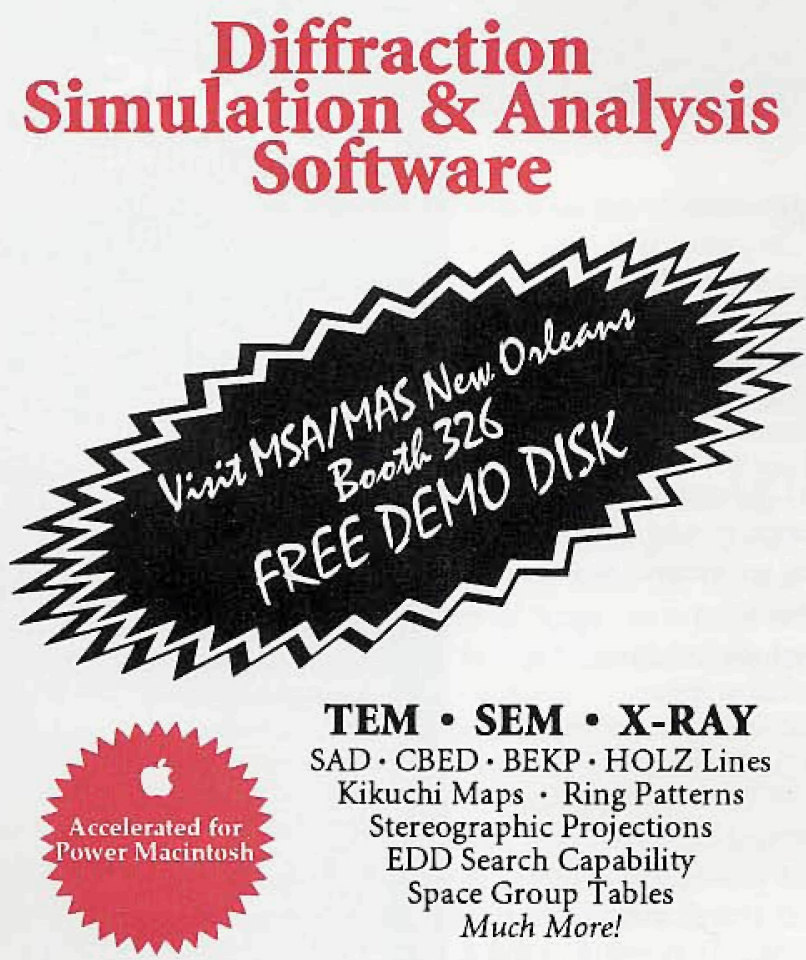

TEM - SEM - X-RAY

$\mathrm{SAD} \cdot \mathrm{CBED} \cdot \mathrm{BEKP} \cdot \mathrm{HOLZ}$ Lines

Kikuchi Maps - Ring Patterns

Stereographic Projections

EDD Search Capability

Space Group Tables

Much More!

VIRTUAL LABORATORIES

P.O. Box 14266. Albuquerque, NM 87191 USA

tel: 505-828-1640 - fax: 505-822-9759 resolution which can be achieved is about 1.75 microns.

The image formation process is very simple. The lens focusses to a point on the specimen equal in size to approximately its wavelength. At each point of insonification the reflected signal is envelope detected. The amplitude of the resultant pulse is assigned a relative gray scale value from 0 to 255 where 0 corresponds to black and 255 to white. In this manner, a gray scale image is generated which is quantitatively representative of the interaction of sound with the specimen under investigation. The largest two dimensional image scan possible with SAM at frequencies above $100 \mathrm{MHz}$ is $2 \mathrm{~mm}$ square. The smallest scan corresponds to $0.1 \mathrm{~mm}$ square. For each possible scan width, there are $640 \times 500$ pixels of information collected. Magnification, as such, is not the same concept with SAM as it is defined in optics. Magnification in scanning acoustical microscopy is the ratio of the size of the image displayed on the video monitor to the size of the scan. In the case of a $0.1 \mathrm{~mm}$ scan displayed on a $24 \mathrm{~cm}$ video screen, the magnification factor is 2400 .

So what does this scope have over others which are well established in the market? For one, acoustical waves interact with specimens in quite a different manner. Acoustical waves can penetrate a specimen non-destructively or noninvasively. This results in being able to visualize structures internally. Because this a reflection microscope, an acoustical pulse is emitted by the transducer-lens system. The sound wave is transmitted through the specimen by means of a liquid coupling solution. The coupling medium is usually distilled water, but practically any liquid will do as long as the gating of the pulses is manually set. SAM is set-up automatically for water as the interface between the lens and the specimen. Whenever a change in impedance of the specimen is detected, a reflection occurs. For those of you unfamiliar with the term impedance, consider it equivalent to resistance. Acoustical waves travel through a specimen without resistance until a region where a change in impedance is encountered. At that time, part of the incoming wave will be reflected back towards the lens, the remaining part will continue forward until another such region is encountered. In this way, this process makes it possible to detect delaminations imbedded within materials.

In the case of biological materials, specimens can be prepared in the same manner as for optical visualization up to the point of staining. No staining of the specimen is required for acoustical imaging. Since biological specimens are mainly composed of water, the image formation process (ie the contrast necessary to be able to differentiate between structures) will be based on the amount of absorption and scattering of the acoustical waves. Therefore, the image produced can be directly related to a quantitative acoustical parameter which is called attenuation. Attenuation, in acoustics, is the sum of the contributions from the absorption and scattering components. Unfortunately, as the frequency of the lens increases, attenuation of sound waves also increases quite rapidly. This means that only surface imaging is possible at the higher frequencies. As the frequency decreases, more depth penetration in the specimen is possible. Subsurface evaluations can then be attempted. This applies to biomaterials as well. The optimal biological specimen for high frequency visualization is between $5-10$ microns in thickness, air-dried on a glass (or better yet a sapphire) slide. The glass slide (or sapphire) act as a perfect specular reflector. The acquired image is then a tue representation of attenuation indicated by the resultant gray scale. Measurements of elasticity can

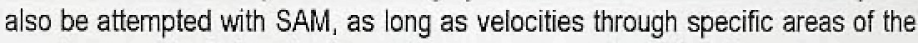
specimen can be determined as well as measurements of density.

This discussion only briefly attempts to demonstrate the immense capabilities and versatily of acoustical microscopy. The next article will explain in more detail some very specific applications of SAM where measurements such as attenuation and elasticity are parameters important to have some insight into.

The author would very much like to receive comments or questions (e-mail: jgallet@orion.oac.uci.edu).

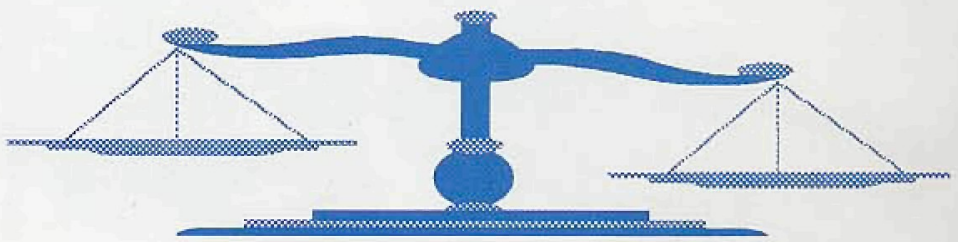




\section{REQUEST FOR BIDS:}

\section{No Reasonable Offer Refused!!!}

\section{Cryofixation System \& Molecular Distillation Systems}

Interested parties are invited to submit offers to purchase the following NEW systems:

LifeCell CF 100 Cryofixation System. Based on the technique of metal mirror cryofixation, the CF 100 operates by rapidly applying a tissue sample to the mirror-finished surface of a gold bonded, pure copper block, cooled by continuous liquid nitrogen flow. All parameters of the system, including sample holder design, plunger delivery, cryogenic chamber conditions, and efficiency of thermal sink have been optimized to produce quality cryofixation. A programmable microprocessor directs each parameter to its optimum value, so that same quality is reproduced for each sample. Original list price: $\$ 27,500$.

LifeCell MDD-C Molecular Distillation Dryer. The molecular distillation dryer allows cryofixed samples to be heated at a calculated rate which, in conjunction with the ultra-high vacuum and efficient condensing surfaces dries the cryofixed sample in a dynamic manner. Once dried, the samples can be stabilized with a vapor fixative and then infiltrated with a resin. Original list price: $\$ 87,450$.

These new items are available without manufacturer's warranty. Philips reserves the right to reject any or all bids. Bids are due in writing to the address below by 12:00 noon on August 15, 1994. For additional information or brochures contact:

Philips Electronic Instruments Co.
Electron Optics Marketing
85 McKee Drive
Mahwah, NJ 07430

Tel.: (201)529-6165

Fax: (201)529-2252

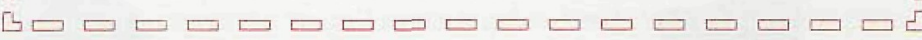

FREE

Electro Magnetic Field

FREE

GAUSSMETER

with purchase of four scintillators

(Retail only)

Meter also for sale at $\$ 59.95$ each

Magnetic Fields affect SEM's, TEM's \& your health

M.E. TAYLOR ENGINEERING INC.

21604 Gentry Lane - Brookeville, MD 20833

Tel:: (301)774-6246 - Fax: (301)774-6711

\section{MICROSCOPES AREN'T JUST FOR MICROSCOPISTS, ANYMORE! \\ Stephen W. Carmichael, ${ }^{1}$ Mayo Clinic}

Historically, microscopes have been used to gather morphologic data. We have called people who use these instruments microscopists, and it is implied that microscopists are morphologists. As was pointed out in the April/May issue of this newsletter, useful information about a specimen is also gained from temporal analysis. Further, it has been appreciated that the new family of scanning probe microscopes can be used to gather additional types of information so that these instruments have the potential to be useful beyond the dreams of a conventional microscopist. As we will discuss in this article, the future is here for one such application.

The atomic force microscope (AFM) takes advantage of the leverage afforded by the deflection of a laser beam bounced off a cantilevered stylus that is scanned over the surface of a specimen. As the stylus is deflected by a small change in the topography of the surface, the light beam begins to be deflected away from a photodetector. Piezoelectric devices in the arm suspending the cantilever correct the position of the stylus so that the light beam remains on the photodetector. A computer can then use the amount of "correction" utilized to reconstruct a map of the scanned surface with incredible resolution.

But what of the forces of attraction or repulsion between the stylus and the surface? Can these forces be measured? Could this be useful information? Can this be utilized to quantitate binding forces between biologic molecules? No doubt these questions have been asked by several "microscopists" using the AFM, but Ernst-Ludwig Florin, Vincent Moy, and Hermann Gaub appear to be the first to directly measure the forces binding two protein molecules. ${ }^{2}$ They coated a conventional silicon nitride stylus with bovine serum albumin, attached biotin, and covered the biotin with avidin. The functionalized tip was then advanced to an agarose bead that had been covered with biotin. As the tip made contact with the bead, the cantilever was deflected. The stylus was then retracted from the bead and a deflection in the opposite direction (this was caused by the attraction between the biotin and avidin) was detected until the bonds between the molecules were disrupted and the cantilever sprang back to the neutral position. This deflection could be directly converted to force because the spring constant of the cantilever was known. Florin et al. presented convincing evidence that the force required to separate a single biotin molecule from avidin is $160 \pm 20 \mathrm{pi}$ conewtons ( $\mathrm{pico}=10^{-12}$ ). This is an impressive feat that apparently constitutes a novel application of the AFM.

Florin et al. go on to suggest that with modifications this technique may be used to directly measure ligand-receptor forces on the surface of a living cell. This would be remarkable, indeed! There are clearly some major problems to be overcome before this would be possible. A comparison of binding constants suggests that the forces involved in drug-receptor and antigenantibody interactions are about three orders of magnitude lower than the biotin-avidin binding measured in this study. Difficulties created by the "springy" surface of a cell and noise imposed by thermal fluctuations (Brownian motion) are not trivial. Another method that may better measure such small forces in a living system may come from a related application of "optical-force microscopy." As demonstrated by Lucien Ghislain and Watt Webb, ${ }^{3}$ the spring constant of an optical-force transducer is comparable to the mechanical cantilever of the AFM. It is probably just a matter of time before "microscopists" are clever enough to exploit this feature to directly measure forces between molecules.

It is evident that microscopes are moving out of their traditional roles of depicting morphology. The ability to directly measure biologic forces has been added to the armamentarium of the modern microscopist.

The author gratefully acknowledges the help of Vincent Moy. Technical University of Munich, and David Clapham, Mayo Clinic, and the members of his laboratory. 2 Florin, E.-L., V.T. Moy, and H.E Gaub, Adhesion forces between individual ligand-receptor pairs, Science 264:415-417, 1994.

3. Ghislain, L.P., and W.W. Webb, Scanning-force microscope based on an optical trap, Optics Letters 18:1678-1680, 1993 